\title{
Rétention de différentes espèces microbiennes lors de l'épuration du lait par microfiltration en flux tangentiel *
}

\author{
E Trouvé ${ }^{1 * *}$, JL Maubois ${ }^{1}, \mathrm{M}$ Piot ${ }^{1}, \mathrm{MN}$ Madec ${ }^{1}, \mathrm{~J}$ Fauquant ${ }^{1}$, \\ A Rouault 1, J Tabard 2, G Brinkman 3 \\ 1 INRA laboratoire de recherches de technologie laitière, 65, rue de St-Brieuc, \\ 35042 Rennes Cédex; \\ 2 Société Alfa-Laval, 78340 Les Clayes-sous-Bois; \\ ${ }^{3}$ Société des céramiques techniques, 65001 Tarbes, France
}

(Reçu le 2 août 1990 ; accepté le 26 novembre 1990)

\begin{abstract}
Résumé - La rétention de 7 espèces bactériennes représentatives de la flore contaminante des laits crus par les membranes de microfiltration mises en œuvre dans le procédé Bactocatch a été étudiée. Ces espèces étaient ensemencées dans 4 fluides laitiers : perméat d'ultrafiltration; microfiltrat de lait obtenu sur membrane $0,2 \mu \mathrm{m}$; solution de phosphocaséinate; lait écrémé pasteurisé.

Les résultats obtenus montrent que le nombre de réductions décimales observé est en moyenne de 2,6 et est indépendant du niveau initial de contamination, ce qui amène à la conclusion que les membranes utilisées dans le procédé fonctionnent comme des filtres en profondeur et non pas comme des filtres écrans. Le taux de rétention des bactéries par la membrane $1,4 \mu \mathrm{m}$ varie avec le volume cellulaire moyen entre $99,93 \%$ et $99,99 \%$. La comparaison des rétentions observées en fonction de la composition des fluides mis en œuvre laisse supposer un effet positif non négligeable des composants solubles du lait, qui interréagissent avec les grains d'alumine composant la membrane avant de favoriser l'adsorption interne des microorganismes.
\end{abstract}

microfiltration / épuration bactérienne / Membrane céramique / lait / qualité bactériologique

Summary - Retention of various microbial species during milk epuration by cross-flow microfiltration. Retention of 7 bacterial species representative of typical contaminant flora of raw milks by microfiltration membranes used for the Bactocatch process was studied. These bacteria were added to 4 dairy liquids: milk ultrafiltrate; milk microfiltrate obtained with $0.2 \mu \mathrm{m}$ membrane; native phosphocaseinate solution and HTST pasteurized skim milk. The results show that the average decimal reduction equals 2.6 and is independent of the initial level of the bacterial population. This indicates that membranes were acting as depth filters and not as screen filters. Bacterial retention rates on 1.4- $\mu \mathrm{m}$ membrane were between 99.93-99.99\%. They were slightly linked to average cellular volume. Comparison of retention observed in function of the biochemical composition of liquids experimented with, led to the conclusion that there is a non-negligible effect of soluble milk components which interact with alumina particles before favorising internal adsorption of microorganisms.

microfiltration / bacteria removal / ceramic membrane / milk / bacteriological quality

\footnotetext{
* Cette étude a été réalisée dans le cadre d'un programme contractuel financé par l'Association Bretagne biotechnologies alimentaires, par la région Bretagne et par le ministère de la Recherche et de la Technologie.

** Correspondance et tirés à part
} 


\section{INTRODUCTION}

L'assainissement des liquides alimentaires et notamment du lait se réalise généralement par application d'un traitement thermique. Selon l'effet bactéricide recherché, le couple temps-température appliqué détruira une proportion convenable de la flore pathogène (pasteurisation) ou de tous les types de microorganismes présents (stérilisation). Mais de ce traitement thermique résultent des modifications irréversibles des composants du lait et de leurs équilibres d'interactions, modifications se traduisant globalement par une diminution de l'aptitude à la transformation fromagère. Aussi a-t-il été recherché d'autres moyens physiques d'élimination des germes contaminants du lait et notamment de leurs formes de résistance : les spores qui sont à l'origine des défauts de conservation des laits pasteurisés de consommation (Bacillus cereus - Bacillus subtilis, etc) ou de qualités organoleptiques en fromagerie de pâtes pressées cuites (Clostridium tyrobutyricum).

L'emploi de la force centrifuge (10 000 g) est une pratique devenue courante dans la plupart des grandes fromageries productrices d'emmental. Mais les équipements utilisés, les bactofugeurs, n'éliminent que $95 \%$ des spores butyriques, ce qui est très insuffisant en cas de moyenne à forte contamination des laits. De plus, une proportion, certes faible, mais non négligeable de la caséine est sédimentée, ce qui abaisse le rendement fromager s'il n'y a pas de réincorporation de ce sédiment après stérilisation (Simonart et Debeer, 1954 ; Bergère, 1987).

L'utilisation de la microfiltration tangentielle sur membrane a été suggérée par Holm et al (1986) et Piot et al (1987). Les progrès accomplis dans la mise au point de nouvelles membranes en céramique ayant un support très perméable et une géométrie multicanaux (Gillot et al, 1984) et la mise en œuvre d'un nouveau concept hydraulique - réalisation d'un co-courant et d'une contrepression dynamique côté microfiltrat aboutissant à une pression transmembranaire uniforme (Sandblom, 1974) ont permis d'accroître l'efficacité de l'opération de séparation particulaire envisagée. II en est résulté les équipements et la technologie qualifiés de "Bactocatch" (Vincens et Tabard, 1988; Malmberg et Holm, 1988; Meersohn, 1989). Ce procédé met en œuvre des membranes en alumine ayant un diamètre nominal moyen de pores de $1,4 \mu \mathrm{m}$. Les performances annoncées sont : un débit de perméation supérieur à $500 \mathrm{I}^{-1} \mathrm{~h}^{-1} \cdot \mathrm{m}^{-2}$ à $50^{\circ} \mathrm{C}$ maintenu pendant au moins $6 \mathrm{~h}$ avec un taux d'élimination des germes microbiens supérieur à $99,5 \%$ (Meersohn, 1989).

La mise à notre disposition dans le cadre d'une collaboration contractuelle d'un équipement pilote Bactocatch nous a permis de vérifier ces performances sur les laits crus de mélange, collectés dans l'Ouest de la France (Trouvé et al, 1989).

Etant donné, d'une part, l'ampleur du champ d'utilisations du procédé Bactocatch en transformation laitière - a priori, son application permettrait une amélioration généralisée de la qualité bactériologique et hygiénique de tous les produits dérivés des laits collectés - et d'autre part, le fait que les mesures de rétention bactérienne par les membranes n'avaient été réalisées que sur des groupes peu définis de bactéries - flore mésophile totale, spores de $B$ cereus ou de $C$ tyrobutyricum (Olesen et Jensen, 1989) - nous nous sommes attachés dans le présent travail à étudier systématiquement la rétention de 7 espèces bactériennes, les plus fréquemment rencontrées dans le lait cru, ajoutées en populations connues à des liquides simulant progressivement le lait écrémé. 


\section{MATÉRIEL ET MÉTHODES}

\section{Equipement utilisé}

L'appareillage mis en oeuvre était la partie micro-filtration d'un équipement pilote Bactocatch. Elle comportait une cartouche 7 P 19 de membranes en alumine Membralox (SCT, Tarbes, France) ayant une longueur de 850 $\mathrm{mm}$, un diamètre interne de $4 \mathrm{~mm}$. Le diamètre moyen des pores était de 1,4 $\mu \mathrm{m}$. La perméabilité à l'eau à $20^{\circ} \mathrm{C}$ était de 1450 l.h-1 $\mathrm{m}^{-2}$. bar ${ }^{-1}$.

Le circuit hydraulique comprenait : une pompe d'alimentation centrifuge ayant une capacité de 30000 I. $\mathrm{h}^{-1}$ et maintenant la pression d'entrée du circuit rétentat à $3,2-3,5$ bars ; une pompe de recirculation du rétentat ayant une capacité de 50000 I. $\mathrm{h}^{-1}$ ce qui assurait une vitesse tangentielle de $7,6 \mathrm{~m} . \mathrm{s}^{-1}$, une pompe de recirculation du microfiltrat ayant une capacité de 20000 I. $h^{-1}$, ce qui permettait, grâce à la présence de billes de polypropylène dans le compartiment microfiltrat de la cartouche, de maintenir la pression transmembranaire à une valeur voisine de 0,55 bars en tout point de la membrane.

\section{Conditions expérimentales}

La température était maintenue à $50^{\circ} \mathrm{C} \pm 1^{\circ} \mathrm{C}$. Les flux d'extraction étaient maintenus constants aux valeurs de $678 \mathrm{I} . \mathrm{h}^{-1} \cdot \mathrm{m}^{-2}$ côté microfiltrat et de $36 \mathrm{I} . \mathrm{h}^{-1} \cdot \mathrm{m}^{-2}$ côté rétentat, soit en facteur de concentration volumique produit entrant / rétentat voisin de 20 . Le microfiltrat et le rétentat étaient recyclés pendant la durée des expérimentations (45 min chacune). Le volume mis en œuvre à chaque essai était de 2001 .

\section{Prélèvement des échantillons}

Les échantillons destinés à l'analyse étaient prélevés à l'aide de seringues stériles de $10 \mathrm{ml}$, mono-usage. Ces seringues étaient implantées dans des septum placés sur la tubulure d'alimentation et sur les boucles de recirculation du rétentat et du microfiltrat. Les prélèvements étaient réalisés à $15,20,25$ et $30 \mathrm{~min}$ après addition des cultures dans le bac d'alimentation ex- cepté pour Citrobacter intermedius dont la thermosensibilité imposait des prélèvements à 2, 5 , 8 et $11 \mathrm{~min}$. Ces prélèvements étaient immédiatement plongés dans la glace. Les dénombrements étaient réalisés aussitôt.

\section{Nettoyage et désinfection de l'équipement}

Les séquences suivantes étaient observées :

- pousse à l'eau tiède $\left(45^{\circ}-50^{\circ} \mathrm{C}\right)$ jusqu'à l'eau claire (environ $10 \mathrm{~min})$;

- nettoyage alcalin avec Ultrasyl 25 (Henkel, Chalons/Marne, France) à 1,5\% : recirculation de $100 \mathrm{I}$ de solution à $60^{\circ} \mathrm{C}$ pendant $20 \mathrm{~min}$;

- rinçage à l'eau tiède $10 \mathrm{~min}$;

- nettoyage acide avec $\mathrm{HNO}_{3}, 0,5 \%$ - recirculation de $100 \mathrm{I}$ de solution à $60^{\circ} \mathrm{C}$ pendant $5 \mathrm{~min}$;

- rinçage à l'eau tiède $10 \mathrm{~min}$.

Lors des expérimentations comportant l'addition de spores de $C$ tyrobutyricum, une étape de désinfection était introduite dans la séquence avant le nettoyage alcalin comme suit :

- les fluides contenant les spores étaient refroidis à $25^{\circ} \mathrm{C}$, additionnés de 750 ppm de $\mathrm{Cl}$ actif (v/v) et recirculés $30 \mathrm{~min}$, ce qui assurait la destruction des spores avant l'évacuation des fluides. On procédait ensuite à la pousse à l'eau tiède comme indiqué ci-dessus.

\section{Fluides mis en œuvre}

Quatre fluides ont été mis en œuvre après ensemencement avec les différentes espèces bactériennes. Leur composition est donnée dans le tableau I.

L'ultrafiltrat était un perméat industriel de lait cru obtenu sur des membranes Carbosep M1 (Techsep, Miribel, France) - seuil de coupure $80000 \mathrm{Da}$ à l'usine Triballat (Noyal-sur-Vilaine, France). Pour pallier d'éventuelles contaminations durant son extraction et son transport, il était systématiquement à nouveau ultrafiltré sur les mêmes membranes et dans les mëmes conditions hydrauliques dans notre halle pilote dans l'heure qui précédait les expérimentations sur équipement Bactocatch. 
Tableau I. Composition des fluides expérimentaux en $\mathrm{g} / \mathrm{kg}$. Composition of experimental solutions.

\begin{tabular}{|c|c|c|c|c|}
\hline & Ultrafiltrat & Microfiltrat & $\begin{array}{l}\text { Phospho- } \\
\text { caséinate }\end{array}$ & $\begin{array}{l}\text { Lait } \\
\text { écrémé }\end{array}$ \\
\hline Matière sèche & 54,8 & 56,9 & 38,2 & 89,5 \\
\hline \multicolumn{5}{|l|}{ Matières azotées } \\
\hline totales & 2,6 & 6,4 & 33,4 & 33,4 \\
\hline Matières azotées & & & & \\
\hline non protéiques & 1,9 & 1,9 & abs & 2,1 \\
\hline \multicolumn{5}{|l|}{ Matières azotées } \\
\hline non caséiques & 2,3 & abs & abs & 7,7 \\
\hline Lactose & 48,8 & 47,1 & abs & 49,5 \\
\hline Cendres & 4,6 & 4,7 & 3,1 & 7,6 \\
\hline Calcium & 0,28 & 0,32 & 1,10 & 1,20 \\
\hline Phosphore & 0,33 & 0,35 & 0,60 & 0,96 \\
\hline $\mathrm{pH}$ & 6,66 & 6,71 & 7,40 & 6,68 \\
\hline
\end{tabular}

Le microfiltrat était obtenu à partir de lait écrémé de grand mélange pasteurisé $15 \mathrm{~s}$ à $72^{\circ} \mathrm{C}$ traité sur un équipement pilote équipé de $6,8 \mathrm{~m}^{2}$ de membranes Carbosep M14 (diamètre de pores : $0,14 \mu \mathrm{m})$ dans les conditions décrites par Fauquant et al (1988).

La solution de phosphocaséinate natif était obtenue par microfiltration de lait écrémé cru, épuré préalablement sur équipement Bactocatch. Le lait ainsi épuré était mis en contact avec des membranes Carbosep M14 dans les conditions décrites par Fauquant et al (1988). $\mathrm{Au}$ facteur de concentration volumique de 3,5 vol d'eau courante filtrée sur filtre en profondeur de $5 \mu \mathrm{m}$ étaient ajoutés par volume de rétentat. La concentration du rétentat était poursuivie jusqu'à FCV 6 . Le rétentat était ensuite redilué jusqu'à obtention d'une teneur en matière azotée totale $(\mathrm{N} \times 6,38)$ proche de $33 \mathrm{~g} / \mathrm{kg}$.

Le $4^{e}$ fluide était un lait écrémé de grand mélange pasteurisé $15 \mathrm{~s}$ à $72{ }^{\circ} \mathrm{C}$ et provenait de la Laiterie Préval (ULN, Montauban de Bretagne, France).

Ces 4 fluides étaient préparés le jour même des expérimentations d'épuration bactérienne. Leur flore mésophile totale (FMT) n'excédait pas $100 \mathrm{UFC} / \mathrm{ml}$ pour les 3 premiers fluides, et $1000 \mathrm{UFC} / \mathrm{ml}$ pour le lait pasteurisé, et leur flore coliforme était toujours inférieure à 1 UFC/ $\mathrm{ml}$.

\section{Souches bactériennes étudiées}

Les caractéristiques des 7 espèces étudiées sont rassemblées dans les tableaux II et III. Le choix de ces 7 espèces a été guidé par la volonté d'étudier la rétention de bactéries Gram ${ }^{+}(5$ espèces) et $\mathrm{Gram}^{-}$(2 espèces), de volume cellulaire faible $\left(<1 \mu \mathrm{m}^{3}\right.$ ) (4 espèces) ou élevé ( 3 espèces), mobiles (2 espèces) ou non (5 espèces) et de différents types morphologiques: bâtonnets (2 esp), sphères (2 espèces), polymorphes (1 espèce) et coccoïdes (2 espèces).

Les espèces non sporulées étaient repiquées à $1 \%(\mathrm{v} / \mathrm{v})$ en tubes de $10 \mathrm{ml}, 72 \mathrm{~h}$ avant les expérimentations. Après $36 \mathrm{~h}$ à $30^{\circ} \mathrm{C}(48 \mathrm{~h}$ à $45^{\circ} \mathrm{C}$ pour Lactobacillus helveticus), ces tubes de préculture servaient à ensemencer à $1 \%(\mathrm{~V} /$ v) 1 I de milieu de culture pour chaque espèce. Ce sont ces cultures qui, après développement dans les mêmes conditions de temps et de température $\left(36 \mathrm{~h}\right.$ à $30^{\circ} \mathrm{C}$ sauf pour $\mathrm{L}$ helveticus $24 \mathrm{~h}$ à $45^{\circ} \mathrm{C}$ ), étaient ajoutées directement aux fluides expérimentaux.

Les spores de $C$ tyrobutyricum étaient ajoutées par apport direct à un niveau élevé $\left(10^{3}\right.$ $10^{5}$ spores par ml) dans le but de permettre un dénombrement aisé sur gélose RCM (Hirsh et Grinsted, 1954). 
Tableau II. Principales caractéristiques des espèces étudiées. Bacterial species tested and their main characteristics.

\begin{tabular}{|c|c|c|c|c|c|}
\hline \multirow[t]{2}{*}{ Espèces } & \multirow{2}{*}{$\begin{array}{l}\text { Groupe } \\
\text { usuel }\end{array}$} & \multicolumn{3}{|c|}{ Volume cellulaire } & \multirow[b]{2}{*}{ Morphologie } \\
\hline & & Gram & $\begin{array}{r}\text { Moyen } \\
\left(e n \mu m^{3}\right)\end{array}$ & Mobilité & \\
\hline $\begin{array}{l}\text { Citrobacter } \\
\text { intermedius }\end{array}$ & $\begin{array}{l}\text { Flore } \\
\text { coliforme }\end{array}$ & - & 4,1 & + & $\begin{array}{l}\text { Bâtonnets } \\
\text { larges }\end{array}$ \\
\hline $\begin{array}{l}\text { Propionibacterium } \\
\text { acidi-propionici }\end{array}$ & $\begin{array}{l}\text { Flore } \\
\text { propionique }\end{array}$ & $\stackrel{+}{(-)}$ & 0,7 & - & Polymorphes \\
\hline $\begin{array}{l}\text { Clostridium } \\
\text { tyrobutyricum }\end{array}$ & $\begin{array}{l}\text { Flore } \\
\text { butyrique }\end{array}$ & + & 1,9 & - & $\begin{array}{l}\text { Spores ovales } \\
\text { (coccoïdes) }\end{array}$ \\
\hline $\begin{array}{l}\text { Streptococcus } \\
\text { thermophilus }\end{array}$ & $\begin{array}{l}\text { Flore } \\
\text { lactique }\end{array}$ & + & 1,5 & - & $\begin{array}{l}\text { Sphères en paires } \\
\text { et en chaînettes }\end{array}$ \\
\hline $\begin{array}{l}\text { Lactobacillus } \\
\text { helveticus }\end{array}$ & $\begin{array}{l}\text { FLore } \\
\text { lactique }\end{array}$ & + & 2,4 & - & Bâtonnets \\
\hline $\begin{array}{l}\text { Pseudomonas } \\
\text { fluorescens }\end{array}$ & $\begin{array}{l}\text { Flore } \\
\text { psychrotrophe }\end{array}$ & - & 0,9 & + & Bâtonnets \\
\hline $\begin{array}{l}\text { Micrococcus } \\
\text { varians }\end{array}$ & $\begin{array}{l}\text { Flore } \\
\text { caséolytique }\end{array}$ & + & 1,2 & - & Sphères \\
\hline
\end{tabular}

\section{Traitement des résultats bactériologiques}

Les 3 variables bactériologiques mesurées ont été :

- Co population bactérienne initiale (circuit d'alimentation du pilote)

- CM population bactérienne du fluide épuré (circuit microfiltrat du pilote)

- $C R$ population de la fraction enrichie en bactéries (circuit rétentat).

Pour une espèce bactérienne et un fluide donnés, les valeurs de $C o, C M$ et $C R$ ont été obtenues en convertissant la moyenne arithmétique des 4 dénombrements (exprimés en log) en nombre de cellules noté UFC/ml ou en nombre de spores / I.

En conformité avec les critères d'évaluation de la destruction des microorganismes d'une part, et en similitude avec les critères biochimiques du transfert membranaire d'autre part, nous avons calculé le nombre de réductions décimales obtenues par microfiltration, appelé «efficacité» et noté $E$ :

$$
E=\log (C o / C M)=\log C o-\log C M
$$

Cette efficacité peut être exprimée en proportion relative avec :

$$
E \%=100 \times\left(1-10^{-E}\right)
$$

Pour chaque expérimentation, nous avons comparé la population dénombrée dans le circuit d'alimentation à celles dénombrées dans les circuits d'extraction du rétentat et du microfiltrat en calculant le rendement (noté $Y$ ) de la microfiltration selon l'expression :

$$
Y=(C R+19 C M) / 20 C o
$$


Tableau III. Milieux utilisés pour la culture et le dénombrement des espèces étudiées. Culture and enumeration media for the bacterial species tested.

\begin{tabular}{|c|c|c|c|}
\hline Espèce & Souche & Milieu de culture & Milieu de dénombrement \\
\hline $\begin{array}{l}\text { Citrobacter } \\
\text { intermedius }\end{array}$ & CNRZ 43 & Bouillon nutritif (Difco) & RVBA (norme FIL 73 A, 1985) \\
\hline $\begin{array}{l}\text { Propionibacterium } \\
\text { acidi-propionici }\end{array}$ & CNRZ 85 & YEL (Hettinga et al, 1968) & YELA (Hettinga et al, 1968) \\
\hline $\begin{array}{l}\text { Clostridium } \\
\text { tyrobutyricum }\end{array}$ & CNRZ 566 & $\begin{array}{l}\text { Spores produites selon } \\
\text { le protocole ITG, } 1982 \\
\text { et conservées dans de } \\
\text { l'eau distillée stérile à } 4{ }^{\circ} \mathrm{C}\end{array}$ & RCMA (Hirsch et Grindsted, 1954) \\
\hline $\begin{array}{l}\text { Streptococcus } \\
\text { thermophilus }\end{array}$ & CNRZ 702 & $\begin{array}{l}\text { M17 (Terzaghi } \\
\text { et Sandine, 1975) }\end{array}$ & M17A (Terzaghi et Sandine, 1975) \\
\hline $\begin{array}{l}\text { Lactobacillus } \\
\text { helveticus }\end{array}$ & ATCC 12046 & MRS (de Man et al, 1960) & MRS (de Man et al, 1960) \\
\hline $\begin{array}{l}\text { Pseudomonas } \\
\text { fluorescens }\end{array}$ & CNRZ 798 & Bouillon nutritif (Difco) & PCA (norme FIL 101, 1981) \\
\hline $\begin{array}{l}\text { Micrococcus } \\
\text { varians }\end{array}$ & ATCC 15306 & Bouillon nutritif (Difco) & $\begin{array}{l}\text { TSA base }+6 \% \mathrm{NaCl} \\
\text { (Lenette et al, 1980) }\end{array}$ \\
\hline
\end{tabular}

qui prend en compte le facteur de concentration volumique de 20 mis en œuvre dans l'équipement Bactocatch.

\section{Analyse chimique des fluides expérimentaux}

Les teneurs en matière sèche étaient déterminées selon la norme FIL $n^{\circ} 21 B$ (1987); celles en matières azotées totales, matières azotées solubles dans le TCA $12 \%$ et à $\mathrm{pH} 4,6$, selon Aschaffenburg et Drewry (1959); celles en cendres, calcium et phosphore, comme indiqué par Brulé et al (1974). Les teneurs en lactose étaient déterminées selon Acton (1977).

\section{RÉSULTATS - DISCUSSION}

\section{Bilans entrées-sorties des populations bactériennes}

Le tableau IV rassemble les résultats obtenus lors du calcul des rendements. Globalement, pour l'ensemble des fluides et des espèces bactériennes expérimentés, environ $1 / 3$ des cellules introduites dans le microfiltre n'est plus dénombré aux 2 sorties de l'équipement. Ce résultat est, en fait, la moyenne de valeurs variant très largement : selon les espèces et les fluides, les 
valeurs du rendement vont de 0,32 (déficit) à 1,12 (excédent). De telles variations sont à rapprocher de l'incertitude des mesures de débit du rétentat qui, faute de régulation

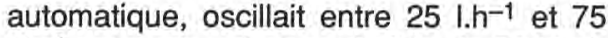
I. $\mathrm{h}^{-1}$ pour une valeur souhaitée de $50 \mathrm{l} . \mathrm{h}^{-1}$, ce qui amenait à des facteurs de concentration volumique (FCV) variant entre 13,5 et 40 et donc à des rendements s'étalant en valeurs instantanées entre 0,68 (déficit) et 1,70 (excédent). À cette incertitude sur la mesure du débit rétentat de l'équipement s'ajoute celle des méthodes de dénombrement bactérien : $\pm 12 \%$ à $\pm 37 \%$ (AFNOR, 1978). Par ailleurs, la thermosensibilité de certaines espèces étudiées n'est pas nulle à $50^{\circ} \mathrm{C}$, température à laquelle était réalisée l'opération de microfiltration et c'est certainement à elle que doit être attribué le rendement inférieur à 0,50 constaté avec $C$ intermedius. Le $D_{50}{ }^{\circ} \mathrm{C}$ de cette espèce appartenant au groupe des bactéries coliformes est, en effet, égal à 8 min (Evans et al, 1970). Mais, même pour les espèces les plus thermorésistantes comme $S$ thermophilus $\left(D_{70}{ }^{\circ} \mathrm{C}=15 \mathrm{~min}\right)$ ou $L$ helveticus $\left(D_{70}{ }^{\circ} \mathrm{C}=30 \mathrm{~min}\right)$ (Frazier, 1967) ou encore les spores de $C$ tyrobuty- ricum, les rendements moyens n'excèdent pas $0,58-0,69$. II est donc très probable qu'une partie des bactéries des fluides, soumis à microfiltration, se fixe soit à la surface externe, soit aux surfaces internes des membranes. Une telle hypothèse s'appuie sur les très importants volumes poreux disponibles au sein de la membrane et de son support $\left(1,4 \times 10^{13} \mu \mathrm{m}^{3}\right.$ et $4,0 \times$ $10^{15} \mu \mathrm{m}^{3}$ respectivement pour la cartouche 7 P 19 utilisée) pour une éventuelle accumulation de microorganismes (Hsieh et al, 1988). À partir d'un volume cellulaire bactérien moyen de $2 \mu \mathrm{m}^{3}$, une rétention intramembranaire de $5 \times 10^{10}$ cellulles/h ne conduirait qu'à une occupation de $5 \%$ du volume poreux de la membrane sensu stricto ou de $0,002 \%$ du volume poreux du support après $7 \mathrm{~h}$ de fonctinnement.

\section{Efficacité de la microfiltration et espèce expérimentée}

Le tableau $V$ rassemble les déterminations effectuées sur les circuits d'alimentation et le tableau VI présente l'efficacité de la mi-

Tableau IV. Rendement de l'épuration bactérienne par microfiltration (hypothèse : $F C V=20)$. $(Y>1$ : bilan excédentaire; $Y<1$ : bilan déficitaire).

1. Citrobacter intermedius. 2. Propionibacterium acidi-propionici. 3. Clostridium tyrobutyricum. 4. Streptococcus thermophilus. 5. Lactobacillus helveticus. 6. Pseudomonas fluorescens. 7. Micrococcus varians.

Efficiency (CR $+19 \mathrm{CM} / \mathrm{Co})$ of bacterial removal by microfiltration on $1.4 \mu \mathrm{m}$ membranes (hypothetical $V C R=20$ ).

\begin{tabular}{lcccccccc}
\hline Espèces & 1 & 2 & 3 & 4 & 5 & 6 & 7 & $\begin{array}{c}\text { Toutes } \\
\text { espèces }\end{array}$ \\
\hline Fluides & & & & & & & & \\
\hline & & & & & & & & \\
\hline Mitrafiltrat & 0,33 & 0,74 & 0,42 & 0,64 & 0,54 & 0,54 & 1,12 & 0,58 \\
Phosphónttrat & 0,39 & 1,12 & 0,82 & 0,66 & 0,65 & 0,65 & 0,32 & 0,62 \\
Lait écrémé & 0,58 & 0,59 & 0,42 & 0,71 & 0,59 & 0,67 & 0,68 & 0,59 \\
Tous milieux & 0,38 & 0,44 & 0,83 & 0,69 & 0,66 & 0,66 & 0,93 & 0,62 \\
\hline
\end{tabular}


Tableau V. Logarithme de la population initiale des fluides à épurer (alimentation); chaque valeur est la moyenne de 4 répétitions.

1. Citrobacter intermedius. 2. Propionibacterium acidi-propionici. 3. Clostridum tyrobutyricum. 4. Streptococcus thermophilus. 5. Lactobacillus helveticus. 6. Pseudomonas fluorescens. 7. Micrococcus varians.

Logarithmic values of bacterial counts in the feed stream circuit (logCo); each number is the average value of 4 repetitions.

\begin{tabular}{lllllllll}
\hline Espèces & 1 & 2 & 3 & 4 & 5 & 6 & 7 & $\begin{array}{c}\text { Toutes } \\
\text { espèces }\end{array}$ \\
\hline Fluides & & & & & & & & \\
\hline Ultrafiltrat & 3,75 & 3,55 & 2,78 & 5,37 & 5,09 & 3,71 & 4,92 & 4,17 \\
Microfiltrat & 6,22 & 5,24 & 3,84 & 6,60 & 5,11 & 6,59 & 4,85 & 5,49 \\
Phosphocaséinate & 5,82 & 6,28 & 4,35 & 5,40 & 4,97 & 4,27 & 5,11 & 5,17 \\
Lait écrémé & 6,13 & 3,46 & 3,79 & 6,03 & 5,77 & 4,47 & 4,72 & 4,91 \\
Tous milieux & 5,48 & 4,63 & 3,69 & 5,85 & 5,24 & 4,76 & 4,90 & 4,93 \\
& & & & & & & & CV=4,7\% \\
\hline
\end{tabular}

crofiltration pour chaque fluide mis en œuvre et pour chaque espèce bactérienne.

Un niveau de contamination voisin de 100000 germes $/ \mathrm{ml}$ a été recherché. La comparaison des populations bactériennes des circuits d'alimentation et d'extraction du microfiltrat, toutes espèces et tous fluides confondus met en évidence (fig 1) l'existence d'une corrélation linéaire entre ces 2 groupes de population. L'équation de la droite de régression s'établit comme suit :

$$
C M=0,93 \times C o-2,26
$$

avec $R^{2}=0,72 ; N=112 ; \sigma_{x, y}=60$

Globalement, la population bactérienne dans le microfiltrat est donc proportionnelle à la population initialement présente dans

Tableau VI. Efficacité de la microfiltration (en nombre de réductions décimales).

1. Citrobacter intermedius. 2. Propionibacterium acidi-propionicii. 3. Clostridium tyrobutyricum. 4. Streptococcus thermophilus. 5. Lactobacillus helveticus. 6. Pseudomonas fluorescens. 7. Micrococcus varians.

Decimal reduction (logCo-log $\mathrm{Cm}$ ).

\begin{tabular}{|c|c|c|c|c|c|c|c|c|}
\hline Fluides Espèces & 1 & 2 & 3 & 4 & 5 & 6 & 7 & $\begin{array}{l}\text { Toutes } \\
\text { espèces }\end{array}$ \\
\hline Ultrafiltrat & 3,35 & 2,33 & 2,77 & 2,10 & 3,96 & 1,86 & 3,49 & 2,84 \\
\hline Microfiltrat & 3,12 & 2,45 & 2,25 & 2,28 & 2,73 & 2,29 & 2,18 & 2,47 \\
\hline Phosphocaséinate & 2,69 & 1,82 & 2,82 & 2,32 & 2,45 & 2,07 & 1,67 & 2,26 \\
\hline Lait écrémé & 3,16 & 2,18 & 3,02 & 3,25 & 3,63 & 2,12 & 2,47 & 2,83 \\
\hline Tous milieux & 3,08 & 2,19 & 2,71 & 2,49 & 3,19 & 2,09 & 2,45 & ${ }^{2,60} C V=10,4 \%$ \\
\hline
\end{tabular}




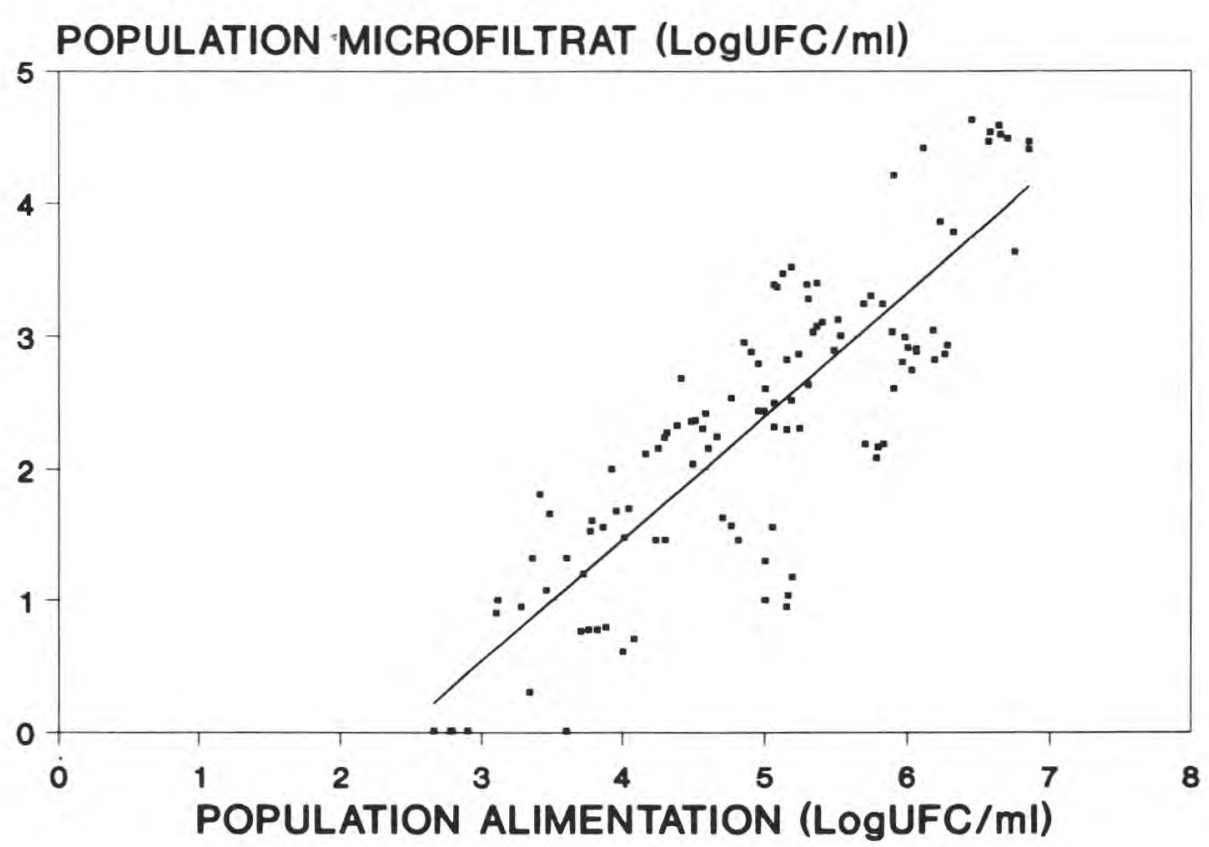

Fig 1. Relation entre populations bactériennes avant (alimentation, log $\mathrm{Co}$ ) et après (microfiltrat, $\left.\log C_{m}\right)$ traitement par microfiltration $(1,4 \mu \mathrm{m})$.

Relationship between bacterial populations in feed stream and microfiltrate.

le fluide traité. Comme pour les autres techniques d'assainissement des liquides alimentaires mettant en œuvre un traitement thermique ou une force centrifuge, le nombre de réductions décimales est indépendant du niveau de population présente dans le fluide mis en contact avec la membrane de microfiltration. La valeur de cette efficacité est en moyenne de 2,6 (99,75\%). II résulte de ces observations que vis-à-vis des bactéries, les membranes de microfiltration utilisées dans le procédé Bactocatch ne fonctionnent pas comme des filtres écrans mais bien comme des filtres en profondeur.

La taille des cellules des différentes espèces bactériennes expérimentées a été évaluée à partir des données du Bergey's Manual et des modèles classiquement retenus pour le calcul du volume cellulaire. Les résultats rassemblés dans la figure 2 indiquent, comme on pouvait s'y attendre, que le taux de rétention bactérien s'élève avec l'accroissement du volume cellulaire. Toutefois, lors de l'épuration bactérienne sur membrane de liquides alimentaires contenant des flores dites sauvages, il est possible que des rétentions non corrélées à la taille cellulaire soient observées. Les bactéries peuvent être agglutinées en amas peu dissociables, les spores peuvent rester adhérentes aux parois de la cellule les ayant formées, des espèces bactériennes particulières peuvent enfin être 


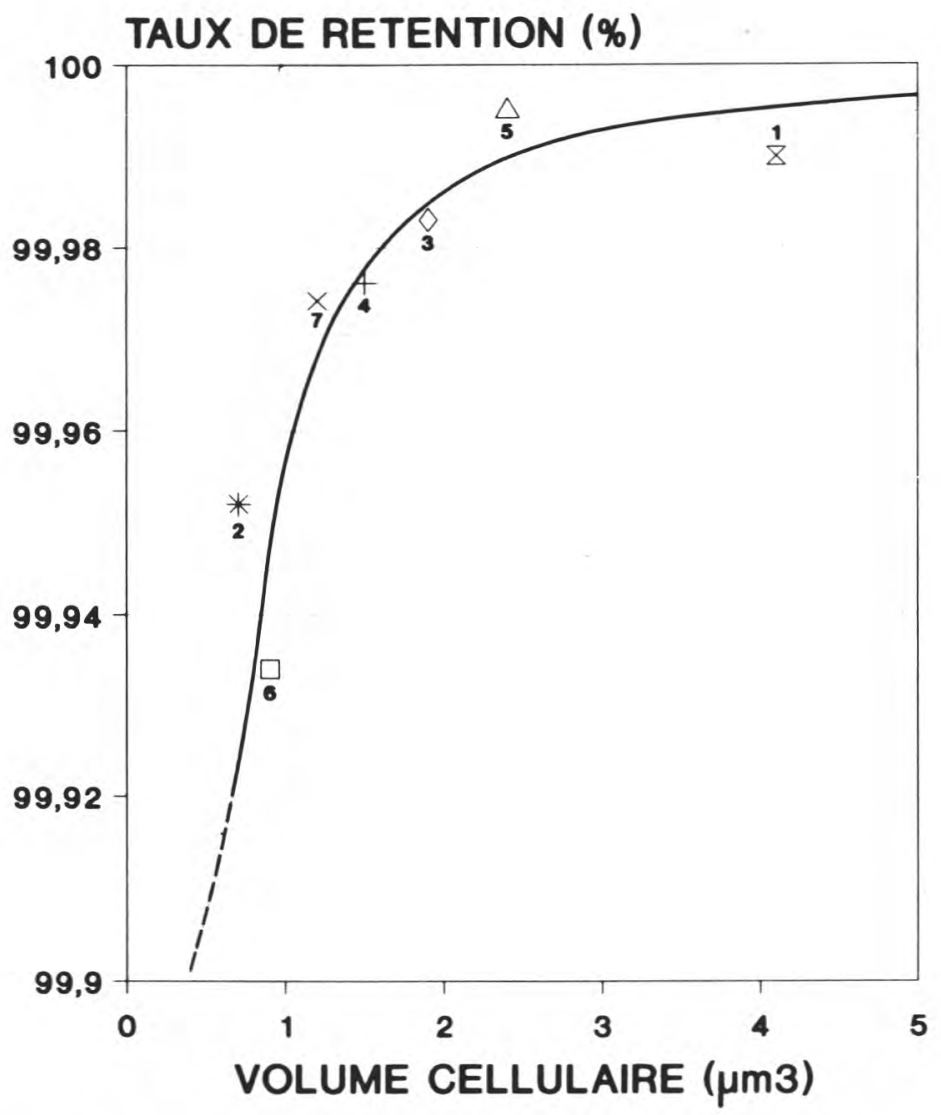

Fig 2. Effet du volume des cellules bactériennes sur le taux de rétention (Bactocatch).

1. Citrobacter intermedius. 2. Propionibacterium acidi-propionici. 3. Clostridium tyrobutyricum. 4. Streptococcus thermophilus. 5. Lactobacillus helveticus. 6. Pseudomonas fluorescens. 7. Micrococcus varians.

Effect of bacterial cells size on retention rate (TR).

étroitement associées à des cellules somatiques.

\section{Efficacité de la microfiltration et fluide expérimenté}

Dans le tableau VII, ont été indiqués pour chaque espèce bactérienne et pour chaque fluide expérimenté, le classement des efficacités de la microfiltration sur les es- pèces bactériennes utilisées lors de nos expérimentations. La somme des rangs fait apparaître que la meilleure efficacité est obtenue pour le lait écrémé, qu'il n'y ait pas ou peu de différence avec l'ultrafiltrat et le microfiltrat mais que, par contre, la solution de phosphocaséinate conduit à la moins bonne rétention bactérienne. Les composants solubles du lait semblent donc jouer un rôle déterminant dans l'épuration bactérienne sur membrane de microfiltra- 
Tableau VII. Rangs de l'efficacité de la microfiltration pour chaque espèce (classement des 4 fluides). 1. Citrobacter intermedius. 2. Propionibacterium acidi-propionici. 3. Clostridium tyrobutyricum. 4. Streptococcus thermophilus. 5. Lactobacillus helveticus. 6. Pseudomonas fluorescens. 7. Micrococcus varians.

Classification of the 4 tested media in order of decreasing logarithmic reduction.

Espèces

Classement des fluides par efficacité décroissante

Ultrafiltrat Microfiltrat Phosphocaséinate Lait écrémé

\begin{tabular}{lrrrr}
\hline & 1 & 3 & 4 & 2 \\
2 & 2 & 1 & 4 & 3 \\
3 & 3 & 4 & 2 & 1 \\
4 & 4 & 3 & 2 & 1 \\
5 & 1 & 3 & 4 & 2 \\
6 & 4 & 1 & 3 & 2 \\
7 & 1 & 3 & 4 & 2 \\
Somme des rangs & 16 & 18 & 23 & 13 \\
\hline
\end{tabular}

tion. Comment ces composants interviennent-ils ? Est-ce par une modification de la réactivité électrochimique de surface des grains d'alumine du fait de leur interaction avec les sels solubles de phosphate de calcium, modification qui initierait la fixation de macromolécules ou d'adsorption suivie de l'adhésion de cellules bactériennes sur et/ou dans la membrane (Coletti-Previero et Previero, 1989) ? La plus faible teneur en phosphates de calcium solubles de la solution de phosphocaséinate expérimentée découlant de la diafiltration réalisée lors de sa préparation serait en bon accord avec cette hypothèse.

Contrairement à ce qui se passe avec les membranes d'ultrafiltration (Maubois, 1980 ), il ne semble pas, au vu des résultats obtenus par Baumy et al (1990) et avec la solution de phosphocaséinate dans cette étude que les phospholipides résiduels du lait ou de ses dérivés interviennent dans un éventuel colmatage irréversible des membranes en alumine de microfiltration.

\section{CONCLUSIONS}

Les résultats obtenus au cours de cette étude prérisent la rétention des espèces bactériennes par les membranes de microfiltration $1,4 \mu \mathrm{m}$ mises en œuvre lors du traitement du lait et de ses dérivés dans le procédé Bactocatch. L'efficacité de la microfiltration est, en moyenne, de $99,75 \%$ (2,6 log) pour les 7 espèces et les 4 fluides expérimentés. Cette valeur s'élève à $99,85 \%(2,83 \log )$ pour le lait écrémé, ce qui est en bon accord avec les données publiées $(99,99 \%)$ par Olesen et Jensen (1989). Cette efficacité est indépendante du niveau initial de population du fluide traité dans la plage étudiée $\left(10^{2}-10^{7}\right.$ UFC/ $\mathrm{ml})$. L'assainissement bactérien réalisé par les membranes de microfiltration est donc, dans son effet, assez semblable à celui réalisé par un traitement thermique mais avec 3 différences fondamentales : la première étant qu'en cas de réalisation d'une seconde microfiltration poussant l'efficacité de la microfiltration à $99,9998 \%$, il n'y aura 
pas d'effet cumulatif dommageable pour les propriétés intrinsèques des macromolécules laitières ou sur leurs équilibres d'interaction, comme cela serait le cas avec les traitements thermiques; la seconde étant que, si le rétentat bactérien traité à ultra haute température n'est pas réincorporé au microfiltrat (Vincens et Tabard, 1988), le lait épuré sur membrane ne contient pas de cadavres bactériens comme tout lait traité thermiquement, et la troisième étant que la thermorésistance des microorganismes n'est pas un critère de sélectivité lors de l'épuration par microfiltration.

Les perspectives ouvertes par l'épuration bactérienne du lait sur membrane sont nombreuses et variées, que ce soit pour la transformation en laits de consommation, ou bien que ce soit pour la transformation en fromages (Vincens et Tabard, 1988; Maubois 1989). Les connaissances à acquérir sont multiples. Devront notamment être précisées l'efficacité de la microfiltration vis-à-vis des espèces bactériennes dangereuses pour la consommation humaine et les conditions dans lesquelles le procédé Bactocatch assure une élimination en proportion compatible avec la santé publique de la flore pathogène et de la flore d'altération (sporulées et non sporulées). Par ailleurs, des protocoles de réensemencement des laits ainsi épurés en vue de leur transformation en différents types de fromage devront être proposés.

\section{RÉFÉRENCES}

Acton GH (1977) The determination of lactose in cheese. Aust J Dairy Technol 9, 111

AFNOR (1978) Directives générales pour le dénombrement des microorganismes. Méthode par comptage des colonies obtenues à $30^{\circ} \mathrm{C}$. Norme NF V 08-011

Aschaffenburg R, Drewry J (1959) New procedure for the routine determination of the va- rious non casein proteins of milk. XVth Int Dairy Congr Londres, 3, 1631-1637

Baumy JJ, Gestin L, Fauquant J , Boyaval E, Maubois JL (1990) Technologie de purification des phospholipides du lactosérum. Process 1047, 29-33

Bergère JL (1987) Élimination des bactéries par centrifugation. In : Le Fromage, (A Eck, ed) Tech et Doc Lavoisier, Paris, 178-181

Brulé G, Maubois JL, Fauquant J (1974) Étude de la teneur en éléments minéraux des produits obtenus lors de l'ultrafiltration du lait sur membrane. Lait 54, 600-615

Coletti-Previero MA, Previero A (1989) Aluminaphosphate complexes for immobilization of biomolecules. Anal Biochem 180, 1-10

de Man JC, Rogosa M, Sharpe ME (1960) A medium for the cultivation of lactobacilli. $J$ Appl Bacteriol 23, 130-135

Evans DA, Hankinson DJ, Kutsky W (1970) Heat resistance of certain pathogenic bacteria in milk using a commercial plate heat exchanger. J Dairy Sci 53, 1659-1665

Fauquant J, Maubois JL, Pierre A (1988) Microfiltration du lait sur membrane minérale. Tech Lait Market 1028, 21-23

FIL (1981) Laits de consommation. Microorganismes psychrotrophes. Dénombrement à $6,5^{\circ} \mathrm{C}$. Norme $n^{\circ} 101$

FIL (1985) Lait et produits laitiers, dénombrement des coliformes. Norme 73A

FIL (1987) Lait, crème et lait concentré non sucré. Matière sèche totale. Norme 21B

Frazier WC (1967) Food microbiology, 2nd ed, Mc Graw Hill Book Co, New York, 88 p

Gillot J, Brinkman G, Garcera D (1984) Nouveaux médias filtrants céramiques pour la microfiltration tangentielle et ultrafiltration. Congrès Filtra, Société Française de Filtration, Paris

Hettinga DM, Vedamuthu ER, Reinbold GW (1968) Pouch method for isolating and enumerating Propionibacteria. J Dairy Sci 51, 1707-1709

Hirsh A, Grinsted E (1954) Methods for the growth and enumeration of anaerobic sporeformers from cheese, with observations on the effect nisin. J Dairy Res 21, 101-110

Holm S, Malmerg R, Svensson K (1986) Method and plant for producing milk with a low bacterial count. Int Patent, PCT no WO 86/01687 
Hsieh HP, Bhave RR, Fleming HL (1988) Microporous alumina membranes. J Membr Sci 39, 221-241

Lenette EH, Balows A, Hausler Jr WJ, Truant JP (1980) Manual of Clinical Microbiology, 3rd edn, Am Soc Microbiology, Washington DC

Malmberg R, Holm S (1988) Producing low bacteria milk by microfiltration. North Eur Food Dairy J 54, 30-32

Maubois JL (1980) Ultrafiltration of whey. J Soc Dairy Technol 33, 55-58

Maubois JL (1989) Applications of membrane techniques in the dairy industry. Proposals for a new IDF group of experts. Bull FIL 214, 26-29

Meersohn M (1989) Nitrate free cheesemaking with Bactocatch. North Eur Food Dairy J 55, 108-113

Olesen N, Jensen F (1989) Microfiltration. The influence of operation parameters on the process. Milchwissenschaft 44, 476-479
Piot M, Vachot JC, Veaux M, Maubois JL, Brinkman GE (1987) Écrémage et épuration bactérienne du lait entier cru par microfiltration sur membrane en flux tangentiel. Tech Lait Market 1016, 42-46

Sandblom RM (Alfa Laval) (1974) Filtering process. Brevet suédois $n^{\circ} 7416257$

Simonart P, Debeer G (1954) Centrifugation et teneur en bactéries du lait. Neth Milk Dairy $J$ 8, 101-106

Terzaghi BE, Sandine WE (1975) Improved medium for lactic streptococci and their bacteriophages. Appl Microbiol 29, 807-813

Trouvé E, Maubois JL, Piot M, Madec MN (1989) Performances de l'équipement pilote MFS 7 Bactocatch sur laits écrémés de grand mélange. CR Bretagne Biotech Alim, Rennes, France

Vincens D, Tabard J (1988) L'élimination des germes microbiens sur membrane de microfiltration. Tech Lait Market 1033, 62-64 